\title{
Guide to Editorial Practice
}

$\mathrm{T}$

HE AIM OF Mark Twain's Letters is to publish, in chronological order, the most reliable and the most legible text possible for every personal and business letter written by or for Samuel L. Clemens, and to publish the letters he received, selectively, as a part of the annotation. The editorial aim for that annotation is to explain whatever in the letters requires explanation, either in notes appended to the letter or, as necessary, in brief narrative passages between letters. The process of establishing the text for each letter is separately documented in the textual commentaries at the back of the volume.

The text for the letters themselves is an inclusive but critical transcription of the original documents sent or, lacking the originals, a critical text established from the most authoritative derived sources available. The goal in either case is a text that is optimally legible and, at the same time, maximally faithful to the text that Clemens himself transmitted. The original documents are therefore emended as little as possible and mainly in order to simplify or omit what would otherwise threaten to make the transcription unreadable or less than fully intelligible in its own right. But when the originals do not survive in an undamaged state, the nonoriginal sources are emended partly for the same reasons, but mainly, and as much as necessary, in order to restore the readings of the original letter, insofar as the evidence permits. In either case, each departure from the source of the text is recorded as an emendation and published in the textual commentary, barring only the most trivial kinds of change, which are not reported item by item, but described briefly at the end of this guide.

Exceptions to this form of the text will occur, but infrequently. For example, two letters that Clemens typed in 1874 (joking the while about his difficulties with the typewriter) clearly exceed the capacity of transcription to capture all their significant details, particularly the typing 
errors to which he alludes in them. Partly because they were typed, however, the original documents are relatively easy to read and therefore can be published in photographic facsimile, preserving most of their details without at the same time making them any harder to read than the originals. But in general, facsimile cannot provide an optimally reliable and readable text, even of Clemens's very legible holograph letters, which comprise at least eight thousand of the approximately ten thousand letters now known to survive. Facsimile does serve to represent within a transcription most elements of a manuscript which would not be rendered more clearly or more faithfully by being transcribed (newspaper clippings, for instance), or that cannot be faithfully transcribed, redrawn, or simulated (drawings, maps, rebuses, to name just a few of the possibilities). Indeed, for a representative handful of letters in each volume, we provide a complete facsimile as a supplement to and a potential test of the transcription, but in no sense as a replacement for it.

The letters have been transcribed using a system of notation and a rationale for emendation which have not before been used to edit letters. We call the result "plain text," in contrast both to "clear text" and its opposite, "genetic text." We require two things of every transcription in plain text: (a) it must be sufficiently faithful to the text of the letter to serve as a reliable substitute for it; and (b) it must be easier to read than the original letter, so long as its reliability is preserved intact. To the extent that maximum fidelity and maximum legibility come into conflict, this way of linking them constrains the pursuit of either virtue at undue expense to the other. But most letters, most of the time, easily satisfy both requirements, yielding a transcription in type which, although clearly not a replacement for the original, can still be read and quoted from as if it were the original.

We have kept the number of newly introduced conventions to a minimum, and for those that are new, we have often adapted familiar conventions of both handwriting and typography in order to transcribe what has tended to be problematic, or else simply ignored, in more traditional kinds of transcription. For instance, to render printed letterhead intelligible at a glance, plain text uses Extra-SMall small capitals for the printed words, and a dotted underscore below whatever Clemens put in the blanks, as for the date and place. And in order to represent all acts of deletion and insertion without making the transcription unreadable, plain text uses line-through cross-outs, "slashes, ${ }^{\phi}$ and ,inferior, carets. 
Most of these devices can now be set with the type itself, making them economical both to set and to print, and many of them can fairly be characterized as type-identical with their handwritten counterparts. A crossout line through type, for instance, needs no interpretation: it simply means deleted, just as it would in manuscript. The effect may be compared with the effect of arbitrary symbols, such as "pointed brackets to the left or right, arrows, bars, and so on"-conventions that are <of necessity $\uparrow$ both $\downarrow$ new and numerous, that may mean something different from one edition to the next, and that must, in any case, be consciously construed at each occurrence. At the same time, the risk of type-identical signs is that their editorial function as signs will be forgotten-that they will seem to picture rather than to transcribe the original manuscript. It is necessary to emphasize, therefore, that despite its greater visual resemblance to the handwritten originals, plain text is emphatically not a type facsimile of them. ${ }^{2}$ Like all diplomatic transcription, and indeed any kind of transcription except type facsimile, plain text does not reproduce the original lineation, pagination, or any nonsemantic aspect of the manuscript unless the writer intended it to bear meaning, which is exactly why it does reproduce many nonsemantic features in the form of typographical conventions that have more or less exact equivalents in manuscript.

Clemens's letters lend themselves to such treatment in part because his training as a printer seems to have given rise to a lifelong fascination with all typographical matters, and in part because he lived at a time when the equivalents between handwriting and type were probably more fully developed and more widely accepted than they had ever been before-or are ever likely to be again. For while, on the one hand, it is clear that Clemens did not intend his private and business letters to be set in type, on the other hand, either by force of habit, or from a certain pride in his expertise, he used the handwritten forms of a great many typographical conventions as consistently and precisely in letters as he did in manuscripts that were intended for the printer. This habitual practice makes it possible to transcribe the letters very much as if they were intended to be set in type-to use the system of equivalents employed by nineteenthcentury writers to communicate precisely with their typesetters-but in reverse, to communicate precisely instead with the modern reader. Clemens's typographical expertise, in short, makes his letters easier to transcribe fully and precisely, as well as to read in transcription, than they otherwise would be, assuming that we understand the meaning of his 
signs and the code for their typographical equivalents exactly as he didan assumption, unfortunately, that cannot always be taken as granted.

\section{The Author's Signs}

A few of the signs to be encountered in these letters may seem unfamiliar, if not wholly exotic. Others may be familiar, even though today they lack the precise and accepted meaning they had when Clemens used them. Especially because some of these signs have fallen into disuse and (partly for that reason) have been adapted by modern editors for their own purposes, it is the more necessary to insist that in our transcriptions of Clemens's letters, they bear only the meaning given them by the writer and his contemporaries. Purely editorial signs in the text are identified on pages xxxviii-xl below, and because some of these purposely adapt typographical conventions, they must be discriminated from authorial signs. But no editorial sign has been used that would entail displacing a normal, easily recognized typographical equivalent for one of the author's signs.

A problem arises, however, precisely because the historical (authorial) meaning of certain handwritten and typographical signs has changed, or become lost altogether. The problem is twofold: how to explicate those signs whose authorial meaning differed from the modern meaning, but can still be recovered, at least in part; and how to represent authorial signs whose earlier typographical equivalent, if any, remains unknown, at least to the editors.

The following Glossary of Special Sorts and table of Emphasis Equivalents are intended to alert the reader to those changes in meaning that we are now able to identify, and to describe the handwritten forms for which the typographical forms are taken to be equivalent-or, in a few cases, for which they have been made equivalent because we lack a better alternative. The glossary includes signs that do not appear in every volume of Mark Twain's Letters, much less in every letter, and doubtless omits some signs that will need to be added in future volumes. Like the glossary, the table of emphasis equivalents provides some information that was, and often still is, regarded as common knowledge. That no table of comparable detail has so far been found in any nineteenth- or twentieth-century grammar, printers' handbook, dictionary, or encyclopedia would appear to indicate that the system of emphasis was almost completely taken for granted, hence rarely made fully explicit even by those who relied upon 
it. At any rate, the particular meaning for Clemens of all such equivalents, including underscores, has had to be deduced or inferred from the letters themselves, and from countless examples of his practice in preparing literary manuscripts for the typist or typesetter (sometimes with the additional evidence of how they responded to his instructions), as well as from the consistent but usually partial testimony found in a variety of printer's handbooks, encyclopedias, manuals of forms, and other documents bearing on what we take to be the system of equivalents between handwriting and type. ${ }^{3}$

\section{Glossary OF SPECIAL SoRTS}

asterisks * * * Always called "stars" by Clemens and by printers generally, asterisks appear in his manuscript as simple crosses $(X)$ or, often when used singly, in a somewhat more elaborate variant of the cross (- $-\dot{*})$. In letters, Clemens used the asterisk as a standard reference mark, either to signal his occasional footnotes, or to refer one part of a letter to another part. He also used asterisks for a kind of ellipsis that was then standard and is still recognizable, and for one now virtually obsolete-the "line of stars"-in which evenly spaced asterisks occupy a line by themselves to indicate a major omission or, for Clemens at any rate, the passage of time. In transcribing the standard ellipsis, we reproduce exactly the number of asterisks in the original, thus: * * * * . In transcribing the line of stars, however, the exact number of asterisks becomes irrelevant, since the device is intended to fill the line, whether in manuscript or in type. The line of stars in our transcriptions is, therefore, always represented by seven asterisks, evenly separated and indented from both margins:

(The conventional order of the standard reference marks was as follows: $*, \dagger, \ddagger, \S, \|, \uparrow$, and, by the end of the century, $\Sigma^{7}$.)

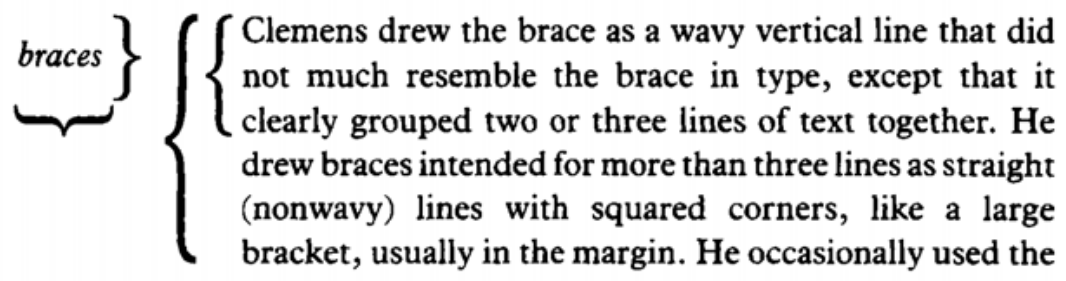


two- and three-line braces in pairs, vertically and horizontally, to box or partly enclose just one line of text. The one-line brace $(\{\})$ was not known to Clemens and would probably have seemed a contradiction in terms. It appears to be a modern invention, but has sometimes proved useful in the transcription when the original lineation cannot be reproduced or readily simulated (see page 219). Otherwise, the transcription prints a brace and preserves, or at least simulates, the original lineation wherever Clemens drew a brace or a nonoriginal copy-text printed one. fist Clemens used the "fist," as it was called by printers (also EI "hand," "index," "index-mark," "mutton-fist," and doubtless other names), not so much as the seventh of the standard reference marks, but for its much commoner purpose of calling special attention to some point in a text. As late as 1871 the American Encyclopedia of Printing characterized the device as used "chiefly in handbills, posters, direction placards, and in newspaper work" (Ringwalt, 217), but Clemens used it often-and without apology-in his letters. We transcribe it by a standard typographical device, either right- or leftpointing, as appropriate, except in special circumstances. For instance, in the following case, Clemens clearly meant to play upon the term "fist" by drawing and using the device as a distinctly open hand:

, 2 "Put it there, Charlie!"

(Envelope of SLC to Charles J. and Olivia L. Langdon, 12 Dec 68, CUMARK.) In all such cases we necessarily reproduce the fist in facsimile, rather than by typographic device.

paragraph If The paragraph sign is both a mark of emphasis and the sixth of the reference marks. It is actually "P" reversed (left for right, and white for black) to distinguish it from that character. Clemens, however, commonly miswrote it as a "P," drawing the hollow stem with large, flat feet, but not the left/right or white/black reversal in the loop. Whenever the sign is used in a letter, we transcribe it by the standard typographical device, with a record of emendation when it has been misdrawn. Clemens used the paragraph sign as a reference mark and as shorthand for the word "paragraph," but most commonly in letters to indicate a change of subject within a passage, one of its earliest meanings. When he inserted the paragraph sign in text intended for a typesetter, he was doubtless specifying paragraph indention. But when he used it in a letter, he was usually invoking the earlier meaning as a substitute 
for indention. The transcription always prints the sign itself, even when it was inserted ( ) or was manifestly an instruction to a typesetter. In the textual commentary, however, the paragraph sign in brackets [ $\rrbracket]$ is editorial shorthand for "paragraph indention."

paraph Clemens drew the paraph, or signature flourish, in a wide variety of forms, from the very simple to the elaborate.

Although nineteenth-century typography certainly used decorative flourishes, a typesetter would not ordinarily transcribe a paraph at all. We do transcribe it, in part because Clemens used the same or similar flourishes apart from his signature, much as a typesetter might use fancy dash rules. In our transcriptions, all flourishes (including paraphs) are rendered by the same arbitrary sign, varied only as necessary to accommodate differing signature lengths.

rules Rules (or rule dashes) in manuscript are usually, but not

$=$ (a) $=$ invariably, centered on a line by themselves, serving to

(b) $=$ separate sections of the text. When used within a line of

(c) - text, they are positioned like an ordinary em dash and may serve as a common form of ellipsis, or simply to fill

blank space in a line. This last function may be compared with the original purpose of the eighteenth-century flourish, namely to prevent forged additions in otherwise blank space. But as with the flourish, this function had in Clemens's day long since dissolved into a mainly decorative one. Rules appear in Clemens's manuscript in three distinguishable species, each with two variant forms. The historical names for the typographical rules appear to be obsolete, even though the rules themselves are still relatively familiar. We construe wavy lines in manuscript as "thick" rules, and straight lines as "thin" rules, regularizing length as necessary. (a) Double rules appear in manuscript as two parallel lines, one wavy and the other straight, in either order. (b) Parallel rules appear in manuscript as two parallel lines, either both wavy or both straight (thick or thin). (c) Plain rules appear as single lines, either wavy or straight (thick or thin).

\section{EMPHASIS EQUIVALENTS}

Clemens used the standard nineteenth-century system of underscoring to indicate emphasis, both relative and absolute, and both within and between words. He indubitably understood the equivalents in type for the various kinds of underscore, but even if he had not, they could prob- 
ably be relied on for the transcription of his underscored words, simply because the handwritten and the typographical systems were mutually translatable. Although we may not understand this system as well as Clemens apparently did, it is still clear that he used it habitually and consistently, and that anomalies are much more likely to result from our, rather than his, ignorance or error.

Occasionally Clemens used what appear to be two variations of a single underscore-a broken underscore (when not prompted by descenders from the underscored word) and a wavy underscore (when more distinctly wavy than normally occurs with any hand-drawn line). If these are in fact variations of a single underscore, they evidently indicate a more deliberate, or a slightly greater, emphasis than single underscore would imply. They have been transcribed in letterspaced italic and boldface type, respectively, even though we do not know what, if any, typographical equivalent existed for them (both are marked ${ }^{\star}$ in the table). Clemens occasionally used letterspacing (with or without hyphens) as an a-l-t-e-r-n-a-t-i-v-e to italic, but he seems not to have combined it with italic, so that this editorial combination always signifies broken underscore. Wavy underscore in manuscript prepared for a printer did mean

\begin{tabular}{|c|c|}
\hline MANUSCRIPT & TYPE \\
\hline lowercase & roman lowercase \\
\hline Capitals and Lowercase & Roman Capitals and Lowercase \\
\hline lowercase & italic lowercase \\
\hline Capitals and Lowercase & Italic Capitals and Lowercase \\
\hline ^Capitals and Lowercase & ${ }^{\star}$ Italic Letterspaced \\
\hline ^Capitals and Lowercase & ${ }^{\star}$ Boldface Capitals and Lowercase \\
\hline lowercase & ROMAN SMALL CAPITALS \\
\hline Capitals and Lowercase & Roman Capitals and Small Capitals \\
\hline CAPITALS or lowercase & ROMAN CAPITALS \\
\hline CAPITALS or lowercase & ITALIC CAPITALS \\
\hline${ }^{\star}$ CAPITALS & ${ }^{\star}$ ITALIC CAPITALS \\
\hline${ }^{\star} \underline{\underline{I}}, \underline{2}, \underline{3}, \underline{4}, \underline{5}$ & ${ }^{\star} \underline{1}, \underline{2}, \underline{3}, \underline{4}, \underline{5}$ \\
\hline
\end{tabular}


boldface, or some other fullface type, at least by 1900 , but it is not clear for how long this convention had been in place. ${ }^{4}$ And in any case, boldface would ordinarily be used for a level of emphasis higher than ROMAN CAPITALS or ITALIC CAPITALS. The use of boldface type to represent wavy underscore is thus an editorial convention.

Clemens also sometimes emphasized capital letters and numerals in ways that appear to exceed the normal limits of the typographical system as we know it. In such cases (also marked ${ }^{\star}$ in the table), we extend the fundamental logic of the underscoring system and simulate one underscore for each manuscript underscore that exceeds the highest known typographical convention. Thus when the pronoun "I" has been underscored twice in manuscript, it is transcribed as an italic capital with one underscore: " $I$ ". Otherwise, underscores in the original letter are simulated only (a) when Clemens included in his letter something he intended to have set in type, in which case his instructions to the typesetter must be reproduced, not construed, if they are to be intelligibly transcribed; and (b) when he deleted his underscore, in which case the transcription simulates it by using the standard manuscript convention for deleting an underscore.

Since underscores in manuscript may be revisions (added as an afterthought, even if not demonstrably so), one virtue of the system of equivalents is that it allows the transcription to encode exactly how the manuscript was marked without resorting to simulation. There are, however, some ambiguities in this reversed use of the code: for example, a word inscribed initially as "Knight" or as "knight" and then underscored three times would in either case appear in type as "KNIGHT." Clemens also sometimes used block or noncursive capitals or small capitals, simulating rather than signing "KNIGHT" or "KNIGHT." Ambiguities of this kind do not affect the final form in the text, but whenever Clemens used block or noncursive letters, or when other uncertainties about the form in the manuscript arise, they are noted or clarified in the record of emendations.

\section{The Author's Revisions and Corrections}

The transcription always represents authorial revisions where they occur in the text, as it does all but the most ephemeral kinds of self-correction. Either kind of change is wholly given in the transcription, except when giving all cases of a phenomenon, or all details of an occurrence, would destroy its legibility. But whenever revision occurs, the transcription in- 
cludes at least the initial and the final reading, with intermediate stages (if any) described in the record of emendations. Self-corrections are emended more frequently than revisions, either to omit or to simplify them, because many could not, for instance, be easily distinguished from revisions except by consulting the textual commentary, even though this distinction is perfectly intelligible in the original letter. Causal evidence in the original, such as a line ending (misspel- -ling) or physical defect resulting in an error, cannot be represented in the text without adding a heavy burden of arbitrary editorial signs. And corrected errors internal to a word are so frequent in manuscript that more than one kind of emendation has had to be invoked to bring their presence in the transcription within manageable, which is to say readable, limits.

The transcription does not distinguish between simple deletions and deletions by superimposition, in which the writer deleted one word by writing another on top of it. Because we have no way to make this distinction legible in the transcription, we represent all deletions as simple deletions, but record as an emendation each instance of deletion by superimposition. For example,

252.11 the str Montgomery - ['Mont' over 'the str']

Since insertions and deletions are always signaled, however, the transcription is almost always as informative as the manuscript about the timing of any change - that is, whether it was made immediately, or on review, or not demonstrably either. Most deletions were immediate, but occasionally the immediacy of a change may be undetectable from the transcription, even though it is clear in the manuscript. For example, "Dont you own" includes a deletion that appears to be either immediate or on review. But the manuscript shows conclusively that Clemens superimposed "you" on "nt," thereby deleting it immediately: he never wrote "Dont you own" but only "Dont" then "Dont you own." The exact timing of some few changes, therefore, can be gathered only from the record of emendations, which in this case reads:

$$
203.12 \text { Dont you - ['y' over 'nt'] }
$$

All deletions that have been transcribed are, perforce, legible to the editors, and were therefore arguably so to the original recipient. But Clemens did occasionally make some deletions easier-or more diffcult-to read than usual. Those obviously intended to be read, or not, are identified in the notes when their special character is not otherwise apparent from the transcription. Deletions by unusual but meaningful 
methods are simulated in the transcription or, if that is unfeasible, described in a note, or both: see page 210 , for instance. But in general, the transcription does not discriminate the various degrees of thoroughness Clemens used in deleting any part of his text. It may be added that some deletions in manuscript, especially of punctuation, were indicated there only by methods that are not themselves transcribable. For instance, when a sentence period has been superseded because Clemens added one or more words to his initial sentence, the initial period has rarely been struck out. Instead, Clemens signaled his intention simply by leaving only the usual word-space between the original last word and his addition, rather than the larger space always left following a sentence period. All such implied deletions are transcribed as if they had been normally deleted, and the fact is recorded as an emendation.

\section{DELETIONS}

- Single characters as words, word fragments, or within words, as well as underscores are deleted with slash marks:

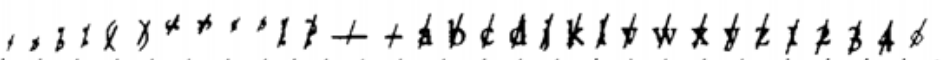

A B

'Annie is well' (66.22)

'I was slow to $d$ take up' (97.24)

' $\angle$ Trip before last' (103.18)

'an absence of $\not 3$ weeks' (77.17)

'sacrifice a feet in that claim' (141.1)

'It reckon you girls' (100.28)

'cheerfullness' (109.22)

- Two or more characters as words, word fragments, or within words are deleted by a horizontal rule:

'the clerks were out in town on business' (108.5)

'had thisca it was well that this calamity' (109.29-30)

'without the greatest interest' (112.17)

- Separate, successive deletions of two or more characters are shown by gaps or breaks in the rule:

'light seeming some alittle sun spreading away' (89.10-11)

These gaps never coincide with line ends in the transcription; rules that continue from the end of one line to the beginning of the next thus always signify continuous deletion, never separate deletions. 
- Deletions within deletions are shown by combining the slash mark and the horizontal rule (for single characters) or by two horizontal rules (for two or more characters):

'then the gallant vessels she bore away to windward.' (352.25-26)

'It was Emma Rowe, Emma Roe, wasn't it?' (248.17-18)

$$
\text { 's }
$$

The earlier of the two deletions is always represented by the shorter line. To read the first stage, mentally peel away the longer line, which undeletes the second stage.

\section{INSERTIONS}

- Single characters inserted between words or within a word:

'voice to a ghastly confidential tone' (303.14-15)

'(when I . . . take Ma to Ky;)'(19.23-24)

'darned if I know which!' (21.20-21)

'and some is a beautiful snowy white' (21.11-12)

'to their room' (304.8)

'It can't be, though.' (100.17)

'said some very startling things' (112.18)

- Two or more characters inserted between words or within a word:

'We (Van. B. \& I,), descended the Ophir incline' (153.24)

'if I hadn't , thoughtlessly , got you into the notion' (157.11-12)

\section{INSERTIONS WITH DELETIONS}

- Insertions with deletions of one or more words, combined in various sequences:

'worth knowing, ${ }_{i}$-the King included, I believe.' (333.9)

'Eighteen months AA short time, ago' (268.11) 'intended to say, Aunt Betsey, that, probably' (94.1)

- Insertions with deletions combined within a word or numeral:

'ThMay-tree' ['may-tree' altered to 'May-tree'] (89.36)

'wishesing' ['es' over 'ing' to make 'wishes'] (66.17)

'necessitary' ['it' over 'ar' of 'necessary' to make 'necessity'] (163.11)

Note that in the above cases the caret indicating insertion is used to identify characters that have usually been superimposed on earlier written characters, thereby deleting them. Superimposition is, in such cases, a 
kind of insertion designed to place new characters next to standing characters: Clemens might have achieved much the same thing, albeit with greater trouble, by literally interlining the characters. The timing of insertions internal to a word must be understood as pertaining only within the sequence of change to that word, not as later than any other part of the text: such changes are, in fact, almost invariably immediate.

Alterations within a word are transcribed in the text only if the original form was a complete word, even though not a possible word in context, or if it was a misspelling or start of a word possible in context. Thus the reader will find 'literafture' (322.20) in the text because it contains the beginning of 'literary', but will not find 'excursinon' (255.19-20) except in the report of emendations because it contains no other word or part of a word possible in context, nor is it a genuine misspelling. This rule of thumb has been invoked because the notation for internally altered words is unconventional, and because such words occur very frequently in manuscript, so that they would pose a serious threat to overall legibility if always transcribed. To further reduce the impediment within manageable limits, we simplify internally altered words, whether or not the original form was a word or start of a word possible in context, whenever Clemens reused three or fewer characters, counting quotation marks, parentheses, dollar signs, and the like. In all such cases we transcribe the initial and final forms as if they had been separately inscribed. Altered numerals are always simplified in this way, even if Clemens reused more than three digits.

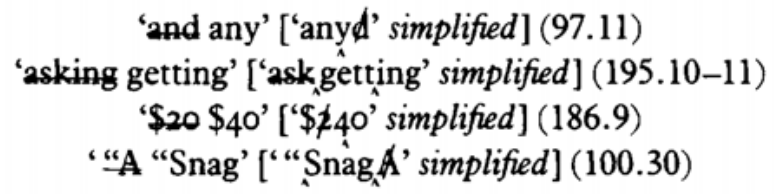

To quote the letters without including the author's alterations, simply omit carets and crossed-out matter, closing up the space left by their omission. For the correct forms of compound words divided at the end of a line in this edition, see the list of emendations for each letter.

\section{The Editors' Signs}

The editorial heading for each letter gives the name of the person or persons addressed, the date or dates of composition, the place or places of composition, as well as joint correspondents, persons writing on Clem- 
ens's behalf, and his amanuensis, if any. A final, separate line in smaller type briefly identifies the source documents used for the text, separated by a colon from their location, usually given as an abbreviation defined in References.

\section{Editorial Heading}

To ... Clemens himself is not named in the heading when he is

From ... the sole author of the letter. When someone else is writing on his behalf, or jointly with him, both Clemens and his agent or coauthor are named in the order of their first appearance in that letter.

per... With documents inscribed or typed for Clemens, the amanuensis is identified following "per."

3 ? October The question mark indicates a conjecture: probably 3 October, but possibly a little later or earlier.

24-29 June A span of dates joined by an en dash indicates a less specific conjecture: the date or dates of composition are thought to fall within this span.

2 and 3 May Not a conjecture, but an assertion that the letter was written in part on each date given.

MS

Manuscript (MS) unmodified in the source line means the transcription has been checked against the original document sent. Modifications in this volume include:

author's copy The source document is in Clemens's hand, but is his copy of the document actually sent.

damage emended The source document is damaged and the transcription includes emendation to restore portions of text no longer visible. See the textual commentary, which usually includes a facsimile of the damaged source.

draft not sent The source document is in Clemens's hand, but is a draft of the document actually sent.

draft telegram The source document is in Clemens's hand, but is a draft of the telegram, not the copy received.

facsimile The source document is a photographic facsimile, published or unpublished, of the original document sent. 
paraphrase The source document is a paraphrase judged to preserve at least some of the original words.

transcript The source document is a printed, handwritten, or typed transcription (omitted when manifestly published).

\section{LETTER TEXT}

VIRGINIA CITY Extra-small small capitals with no initial capitals identify printed text that was not originated by Clemens, such as letterhead or the postmark.

Feb. 19th Dotted underscores signify a blank in the source document, whether or not the blank was so printed in the source, and whether or not Clemens filled it.

Ruled borders are an editorial device to represent the edge of printed or partly printed documents, such as telegram blanks, and are not transcribed from them.

Editorial ellipses, four periods centered in an otherwise blank line, signify an unknown amount of text missing. All other ellipses are transcribed as they appear in the source document.

$\underline{4}_{2}$ emdeletion Deletions are signaled by slash marks through single characters or underscores, and by rules through two or more characters.

making it up. Insertions are signaled by an inferior caret below single characters, and by a pair of carets enclosing two or more characters.

letter text Shaded background identifies text not originated by Clemens, but by someone writing jointly with him. Not used for text originated by Clemens but written in another hand, or text of any origin transcribed below the envelope rule.

[ ] Clemens's brackets are rendered in this form to avoid any confusion with editorial brackets.

[ ] Editorial brackets enclose [editorial description, which is always italicized]; or text omitted inadvertently by the writer an[d] here interpolated by [the] editors; or text modified by description [in margin: All well]. 
$\mathrm{d} \diamond \circ \mathrm{m} \diamond \mathrm{nd}$ The diamond is an editorial sign representing any alphabetical character, numeral, or punctuation mark which the editors cannot read or reliably conjecture. It never signifies word-space. When the number of unread characters cannot be estimated, the problematic text is described, as in [about three words illegible].

letter ${ }^{1}$ text. $^{2}$ Superscript numbers signal editorial notes, which follow the letter itself.

$\mathrm{Sam}^{l} \quad$ Superscript ell is always rendered italic to prevent confusion between superscript one $\left({ }^{1}\right)$ and superscript ell $\left({ }^{1}\right)$.

The envelope and full-measure rule indicate that all text transcribed below them was written or printed on the envelope at the time of transmission, or on the letter itself as an address, receiver's endorsement, or docket.

The vertical rule signifies the end of a line in the source document. It is used only below the envelope rule, in notes, and in the textual commentary.

\section{Emendation of the Copy-Text}

We emend original documents as little as possible, and nonoriginal documents as much as necessary, but basically for only two reasons: to prevent the transcription from including an error, ambiguity, or puzzle that either (a) is not in the original or (b) is in the original but cannot be intelligibly transcribed without correcting, resolving, or simplifying it.

With the system of notation used in plain text, it is technically feasible to transcribe much more detail than is consistent with maximum legibility. For example, the two words 'yourself about' (17.9) might have been transcribed 'yourseflf abotut', as indeed they are in the record of emendations, which reports the omission from the transcription of these two self-corrections. But if the transcription were to include all such selfcorrections, instead of only those permitted by the several rules of thumb already described, their sheer number, in combination with this still unconventional notation, would seriously impair the overall legibility of the letters as a whole. This result is typical, and it means that the crucial factor in deciding whether or not to emend is usually not whether includ- 
ing this or that detail would make the text more reliable or complete (it would), but whether the detail can be intelligibly and consistently transcribed. By and large, if it can, it is, and if it cannot, it is emended.

This practical criterion for deciding "what to put in and what to leave out" does not, however, tell a reader exactly what to expect. Indeed, the more customary way to discriminate between transcribed and untranscribed details is to say, or imply, that the omitted details are not "significant." But there is, in fact, no necessary or obvious hypothetical limit on which details have "significance" in the text of personal letters, and we must assume that almost any semantic or nonsemantic detail of a letter might be "significant" to someone, in some circumstances.

But if there is no clear hypothetical limit on what to transcribe, there is a practical limit, which is jointly determined by the system of notation used and the purpose for which transcription is undertaken in the first place. Since our system of notation can represent more detail than can be made fully legible, nothing would be easier than to produce transcriptions that were more difficult to read than the original letters. But we assume that for most readers, most of the time-including readers interested in these ephemeral details of the original-the basic purpose of transcription is to make the letters easier to read than they are in the widely dispersed original documents. This is not to say that the criterion of legibility amounts to a magic wand, automatically producing reliable texts because they are made legible: with a less flexible system of notation, legibility most surely would not guarantee reliability. Legibility has become the decisive factor for plain text only because its system of notation is capable of including more, and doing so more legibly, than any other system known to us (not to say that it is beyond improvement). But whether or not plain text succeeds in omitting nothing of consequence from the transcription, and nothing that may be of consequence from the record of emendation, is a question that only experience with the result can answer satisfactorily. Meanwhile, it may be helpful at least to survey some recognizable categories of detail that are in fact frequently or invariably emended.

Authorial errors are not emended if they can be intelligibly transcribed. Some few errors are corrected within brackets-specifically those that can be fully and decisively repaired by interpolating what the writer has inadvertently omitted. Such interpolations may be necessary to construe the text at all, let alone to read it easily, and they can coexist with an 
otherwise uncorrected text because they correct errors without concealing them. Strictly speaking, interpolations are not emendations, because like superscript numbers for notes they are always recognizably editorial from the text alone. Interpolations are therefore not routinely recorded as emendations.

Errors in a nonoriginal copy-text, such as a newspaper printing, are always emended when the odds favor even a less than certain recovery of the reading in the lost original. Clemens's precise, even finicky habits make it more, rather than less, likely that errors in such a printing are the typesetter's, especially since the typesetter was in general not committed to an exact transcription but rather to a corrected form of the document being set in type. If an error in a newspaper text is deemed typical or characteristic of Clemens, however, it is not emended, although if it is a simple omission it may be corrected by interpolation. Likewise, when a nonoriginal copy-text seems to depart from the original (for example, in a signature printed as "CLEMENS"), no emendation would be made unless the editors also had substantial evidence of how the signature was in fact written. In short, whenever the text depends upon nonoriginal documents derived from the lost originals, the editors emend the copytext when reliable evidence suggests not just that its reading is mistaken, but also what the likely reading of the original really was.

Damaged texts (usually, but not necessarily, the original manuscripts) are likewise emended whenever possible to restore the original, though now invisible, reading. Emendation in such cases is, despite some appearance to the contrary, still based on documentary evidence: sometimes a copy of the original made before it was damaged, or damaged to its present extent-but more commonly evidence still in the original documents, such as fragments of the original characters, the size and shape of the missing pieces, the regularity of inscribed characters (or type) and of margin formation, the grammar and syntax of a partly missing sentence, and, more generally, Clemens's documented habits of spelling, punctuation, and diction. We undertake such emendations even though they are inevitably conjectural, in part because the alternative is to render the text even less complete than it is in the damaged original (since sentence fragments are unintelligible without some conjecture, however tentative, about the whole sentence), and in part because only a definite, albeit uncertain, conjecture is likely to elicit any effort to improve upon what the editors have been able to perform. For this same reason, a fac- 
simile of any seriously damaged document is always provided, either in an appendix or in the textual commentary.

Alterations in the manuscript, meaning both revisions and selfcorrections, are fully represented in the transcription, except when they are emended because they are too complicated to be readily intelligible in transcription; or because they are self-corrections (corrected false starts, miswritten characters, and the like) which cannot be transcribed intelligibly as corrections; or because one or another kind of detail, such as deletion by superimposition, is not yet susceptible of clear transcription.

End-line occurrences requiring some adjustment in transcription seem nearly infinite in their variety, and are probably the most common occasion for emendation. For example, all ambiguously hyphenated compounds ("water-|wheel") must be rendered unambiguously in the transcription ("waterwheel" or "water-wheel"), since their division at a line end cannot be duplicated. Even noncompound words divided at the end of a line may sometimes be ambiguous in ways that cannot be legibly preserved in the transcription: "wit-|ness" in the original must be either "witness" or "witness." Dittography of words or punctuation likewise occurs most frequently at line ends, physical evidence that makes it readily intelligible as an error in the source but that is lost in a transcription which abandons the original lineation. In other words, dittography becomes more difficult to construe readily when it is simply copied, and it is therefore emended. (This decision in turn requires that intralinear dittography also be emended in order not to give a distorted impression of this overall class of error in the original documents.)

One frequent (and frequently misunderstood) end-line occurrence that is always emended occurs in both original and nonoriginal copytexts, although for slightly different reasons: the em dash following terminal punctuation and at a line end (not to be confused with the intralinear period-dash that for some of Clemens's contemporaries, but not for Clemens himself, was quite ordinary terminal punctuation). The perioddash combination in Clemens's manuscript virtually always occurs at a line end, at least until about the mid-1880s, when he seems to have trained himself not to use it at all, probably because typesetters so often misinterpreted it. The typographical use of "period.- End line" probably originated as an inexpensive way to justify a line of type (especially in narrow measure, as for a newspaper) when a sentence ended so near the right margin that there was not room to set both the standard em quad (space) 
after the period and at least the first syllable of the first word in the next sentence. But Clemens used this same device in his manuscript whenever the last word in a sentence fell too near his right margin to permit the normal space and at least the beginning of the next word. The dash indicates that the slightly short line does not portend a new paragraph, but it may also have some familial relation to the eighteenth-century flourish used to prevent forged additions, since it sometimes occurs at the end of short lines that are followed by a new paragraph. Its function may be generally compared to that of a hyphen used at the end of a line to indicate that, even though a word has been divided, it is still to be understood as one word. In any case, whenever a dash in manuscript follows a period (or other clearly terminal punctuation) and also falls at the end of a line, the dash cannot be intelligibly transcribed because its meaning is a function of its position at the end of a short line. The dash is therefore emended and the change recorded, much the way "ob-|vious" would be transcribed, albeit silently, as "obvious." When "period.--End line" occurs in a newspaper or other printing of a lost manuscript letter, it doubtless reflects the typesetter's own use of this method for right justification, and is necessarily emended. And when "period.-Dash" (that is, period-dash within a line) occurs in such a printing, it is almost certainly the result of the typesetter's misunderstanding the convention in Clemens's manuscript, and is likewise emended.

To save space, we transcribe only routine addresses on envelopes by using the vertical rule $(\mid)$ to signify line end; nonroutine text on envelopes is transcribed by most of the same conventions used for the letters themselves. The text of preprinted letterhead is reproduced in ExTRA-SMaLL SMALL CaPITALS, usually in its entirety, but when unusually verbose, only to the extent that Clemens may be said to adopt or refer to it ("I'm down here at the office"). Only substantive omissions from this preprinted, nonauthorial matter are reported as emendations. Whenever Clemens used any of the following typographical conventions in his manuscript, or whenever they occur in nonoriginal copy-texts and are deemed authorial, the transcription reproduces or simulates them: diagonal indention; hanging indention; half-diamond indention; squared indention; text centered on a line; the flush-left paragraph and the half-line of extra space (its collateral convention); text positioned flush-right; quotations set off by quotation marks, indention, reduced space between lines (reduced leading in type), extra space above, below (or both), smaller characters in 
manuscript (type size in nonoriginals), or any combination of these conventions. In Volume 1, normal paragraph indention is standardized at two ems, with variations of one em and three ems often occurring in the same letter.

We silently eliminate minor, presumably unintended variation in the size of all indentions, and we place datelines, salutations, complimentary closings, and signatures in a default position when this is not contradicted by the manuscript. Likewise, unmistakably large variation in the size of indention is treated as deliberate, or as an error, and reproduced or simulated, not corrected or made uniform. Notes which Clemens specifically did not insert within the letter text but wrote instead in its margin are nevertheless transcribed at the most appropriate place within the text, identified by editorial description: [in margin: All well], or [in bottom margin: over]. Regardless of where postscripts are written, they are transcribed in the order they were supposed to be read, as specified by various means in the original, such as "P.P.S." (clearly intended to be read after "P.S."). Postscripts inserted above the beginning of a letter, therefore, are transcribed at the beginning so long as they were inscribed in the same orientation as the letter itself. When written across or at an angle to the main text-a sign they are not to be read before or in conjunction with the text they precede or cross-they are transcribed at the end of the letter. Only changes in writing media are noted where they occur in the text, as in [postscript in pencil:], from which it may also be reliably inferred that all preceding text was in ink. Line endings, page endings, and page numbers are silently omitted from the transcription, but where they affect the text or its emendation, they are given in the record of emendation.

fuly 1987

R. H.H.

${ }^{1}$ According to Fredson Bowers, "General methods of transcription divide neatly in two," which is to say clear text (with supplementary apparatus containing all details of revision) or genetic text (without apparatus because the text itself contains all details of revision). A clear text transcribes the revised form of a manuscript "diplomatically," meaning that the "transcription exactly follows the forms of the manuscript in spelling, punctuation, word-division, italics (for underlining), and capitalization, but not in matters of spacing or in line-division, nor is a facsimile visual presentation of alterations attempted." A genetic text, on the other hand, includes authorial alterations in place "by means of a number of arbitrary symbols like pointed brackets to the left or right, arrows, bars, and so on," with the common result that it is "difficult to read the original text consecutively; [and] it is impossible to read the revised text at all in a coherent sequence" ("Transcription of Manuscripts: The Record of Variants," Studies in Bibliography 
[1976], 29:213-14, 248). Plain text, however, descends from a kind of transcription not mentioned by Bowers, in which the myriad details of a manuscript (particularly details of the author's alterations) are systematically divided between the text and its apparatus, precisely in order to make the text as complete and informative as possible without destroying its legibility (see $N \mathcal{E} f 1,575-84$ ). The practical result of this division is radically improved by adopting a less obtrusive and more readable system of notation than has been used in the past: plain text simultaneously increases both the amount of detail that can be included in the text and its overall legibility.

${ }^{2}$ It is also not a "literal" text, even though it is probably as inclusive as most texts for which that claim is made. Nor is it, strictly speaking, a "noncritical" text, as defined by G. Thomas Tanselle, since even though it "aims at reproducing a given earlier text [the original letter] as exactly as possible," the editor defines what is possible by what he is able to transcribe legibly. He is therefore "making decisions about how the new text will differ from the text in the document," and the result is necessarily a text that "then becomes a critical text" " Textual Scholarship" in Introduction to Scholarship in Modern Languages and Literatures, edited by Joseph Gibaldi [New York: Modern Language Association, 1981], 32, 47).

${ }^{3}$ This is not the place for a full bibliography of relevant sources, but it may be helpful to name at least those found most frequently useful and pertinent: Thomas F. Adams, Typographia; or, the Printer's Instructor(Philadelphia: L. Johnson and Co., 1857; copyrighted 1845); Theodore Low De Vinne, Correct Composition, 3d ed. (New York: Century Company, 1910; copyrighted 1901); [George A. Gaskell], Gaskell's American Manual and Compendium of Forms, new and revised edition by Loomis T. Palmer (Chicago: M. A. Donohue and Co., 1903); Thomas MacKellar, The American Printer: A Manual of Typography, facsimile of the "fifteenth edition-revised and enlarged" (Philadelphia: MacKellar, Smiths and Jordan, 1885; facsimile by Harold A. Berliner, Nevada City, Calif., 1977); Lindley Murray, An English Grammar: Comprehending the Principles and Rules of the Language . . . In Two Volumes (New York: Collins and Perkins, 1810); [Wesley Washington Pasko], American Dictionary of Printing and Bookmaking (New York: Howard Lockwood and Co., 1894; facsimile edition, Detroit: Gale Research Company, 1967); J. Luther Ringwalt, ed., American Encyclopaedia of Printing (Philadelphia: Menamim and Ringwalt, J. B. Lippincott and Co., 1871); A. A. Stewart, The Printer's Dictionary of Technical Terms (Boston: School of Printing, North End Union, 1912); and C. Stower, The Printer's Grammar; or, Introduction to the Art of Printing (London: B. Crosby and Co., 1808).

${ }^{+}$Citing "Mr. J. Stearns Cushing of the Norwood Press" for his information about "underscorings for display in school-books," including wavy underscore for boldface, De Vinne demonstrates that the convention was well established when he first copyrighted Correct Composition in 1901 (De Vinne, 340). But the date of its origin remains in doubt. 
Letters: 1853-1866 
